SECTION 4. Computer science, computer engineering and automation.

Morozova Tatyana Vladimirovna student of Department. «Radioelectronic systems» Research Institute «Digital signal processing and computer vision»

Don state technical University, Russia

Pismenskova Marina Mikhaylovna student of Department. «Radioelectronic systems» Research Institute «Digital signal processing and computer vision»

Don state technical University, Russia

Voronin Vyacheslav Vladimirovich candidate of technical Sciences, associate Professor,

Department «Radioelectronic systems»

Research Institute «Digital signal processing and computer vision»

Don state technical University, Russia

\title{
ALGORITHM OF SEARCHING FOR SIMILAR BLOCKS IN THE IMAGE WITH THE USE OF TEXTURE ANALYSIS.
}

The purpose of this work is the study of algorithms to find similar blocks in the image with the help of texture analysis.

Keywords: textural analysis, computer vision.

\section{АЛГОРИТМ ПОИСКА ПОХОЖИХ БЛОКОВ НА ИЗОБРАЖЕНИИ С ИСПОЛЬЗОВАНИЕМ ТЕКСТУРНОГО АНАЛИЗА}

Целью данной работы является исследование алгоритмов поиска похожих блоков на изображении с помощью текстурного анализа.

Ключевые слова: текстурный анализ, компьютерное зрение.

Несмотря на стремительный уровень развития большого количества мультимедийных приложений, использующих компьютерное зрение, остается ряд задач, решение которых достаточно проблематично. К их числу относятся задачи распознавания и сопоставления изображений. Данные задачи актуальны и их решение потенциально применимо во многих областях, начиная от регистрации движения и заканчивая поиском дубликатов изображений в базах данных.

Одним из главных этапов во многих задачах компьютерного зрения является поиск похожих блоков на изображениях и кадрах 
видеопоследовательности. Данная задача актуальна в задаче отслеживания траектории движения объектов, поиска дубликатов изображений и т.д.

Упрощенная математическая модель исходного черно-белого изображения $S$, состоящая из двух областей: текстуры и однородной области (рис.1), где $R$ - базовый блок размерностью $\mathrm{m} \times \mathrm{n}$ пикселей для которого находится похожий, а $M$ - блок максимально похожий на $R$ по какому-либо критерию.

Рассмотрим наиболее популярные подходы определения похожих блоков на изображении с помощью текстурного анализа. Понятие текстура является важным пространственным признаком, описывающим распределение цветов или интенсивности яркости в теории обработки двумерных сигналов. Под текстурой понимают специфические повторяющиеся изменения тона (цвета) в изображении объекта или его некоторой части $[2$, c. 947-950]. Текстура может представлять упорядоченное изменение тона в виде геометрически правильных или почти правильных рисунков. Другой тип - стохастическая текстура. Она присуща естественным объектам и, как правило, является следствием шероховатости наблюдаемых объектов.

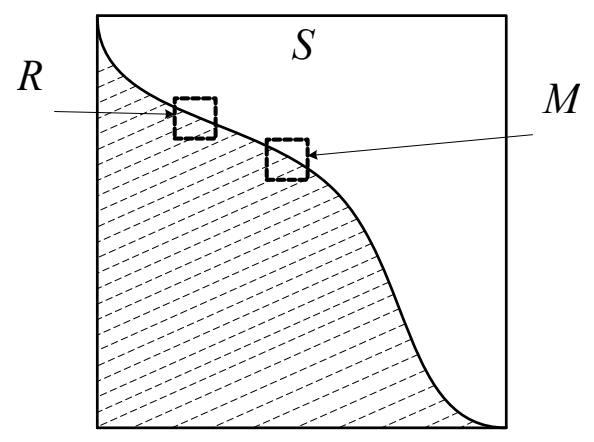

Рисунок 1 - Модель изображения

Лавс (Laws) разработал энергетический подход, в котором оценивается изменение содержания текстуры в пределах окна фиксированного размера [1, с. 1264-1269]. Для вычисления энергетических характеристик используется набор из 22 масок размерами $5 \times 5$. Затем энергетические характеристики каждого пикселя анализируемого изображения представляются в виде вектора и 9 чисел. Для вычисления масок используется следующие векторы: L5 (Level) = [1 4664 1], E5 (Edge)

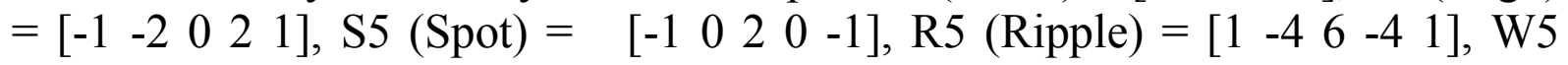

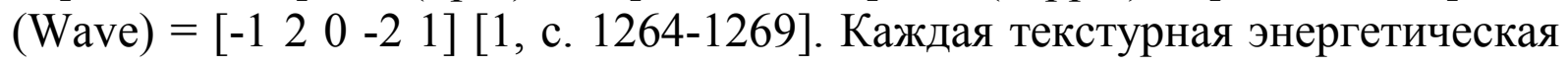
карта является полноразмерным изображением, которое представляет результаты обработки входного изображения с использованием $k$-й маски [3, с. 87]. Таким образом, используя фильтры Лавса, для любого пикселя получаем описание характеристик текстуры в некоторой окрестности выбранного пикселя. Соответственно представляет интерес поиск похожих 
блоков на каждой энергетической карте, при этом комбинацию найденных блоков в дальнейшем можно использовать для синтеза наиболее похожего блока.

Вторым подходом к описанию текстур является использование фильтра Габора, который представляет собой линейный фильтр, импульсная характеристика, которого определяется в виде гармонической функции, умноженной на гауссиан. Этот фильтр широко применяется для распознавания границ объектов и выявления направления текстуры. В результате применения данного ядра с различными параметрами $\theta=0$, $\pi / 4, \pi / 2,3 \pi / 4$, и масштабами: $5 * 5,10 * 10,20 * 20,40 * 40$ получается 16 массивов $[4$, с. 229$]$.

После применения фильтра Габора для каждого пикселя изображения получается вектор признаков, который эффективно описывает локальную текстуру окрестности. Фильтры Габора обладают геометрической устойчивостью, а именно, они устойчивы к операциям масштабирования, поворота, изменения яркости или контрастности.

Предлагается, так же, как и в случае Лавса использовать поиск на каждом полученном изображении после сверти с ядром Габора при различных параметрах. В качестве меры близости используется среднеквадратичное отклонение заданного блока $\mathrm{R}$ с каждым блоком M изображения.

Для исследования сравнения эффективности предложенных выше подходов поиска похожих блоков на изображении с помощью текстурного анализа было выбрано тестовое изображение Square из библиотеки Kodac. Оценка эффективности качества обработки изображений проводится на основе субъективного критерия сравнения.

На рисунках 2 и 3 представлены результаты поиска блока $R$ для случая, когда блок выбран на слабоконтрастной области. Визуальный анализ результатов показывает, что представленные маски Laws определяют похожий блок достаточно точно (а - маска R5W5/W5R; б маска E5E5; в - маска E5R5/R5E5; г - маска S5S5).

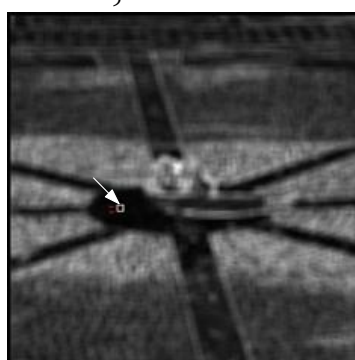

a)

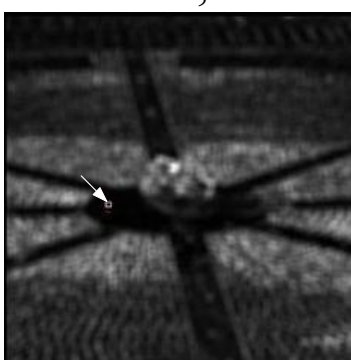

б)

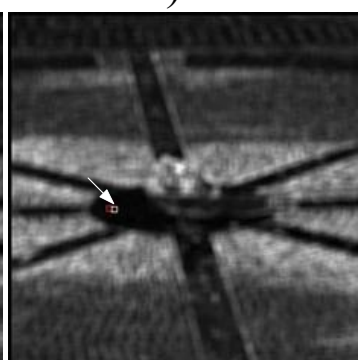

B)

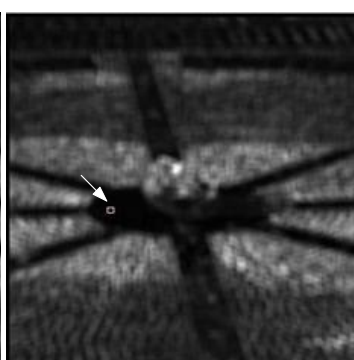

г)

Рисунок 2- Результаты обработки тестового изображения Square: a) маска R5W5/W5R; б) маска E5E5; в) маска E5R5/R5E5; г) маска $\mathrm{S5S5}$ 
Аналогичные результаты получены для фильтров Габора (рис. 3).

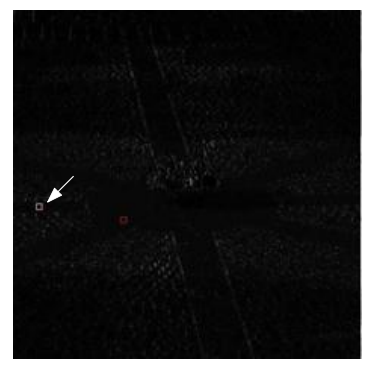

a)

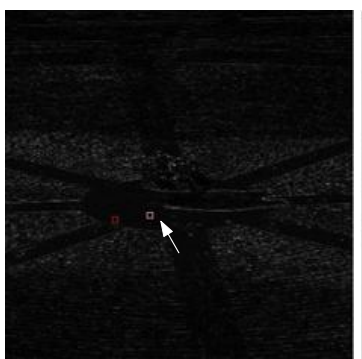

б)

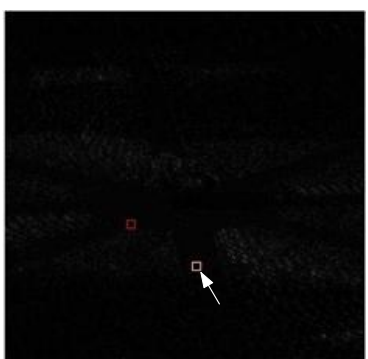

B)

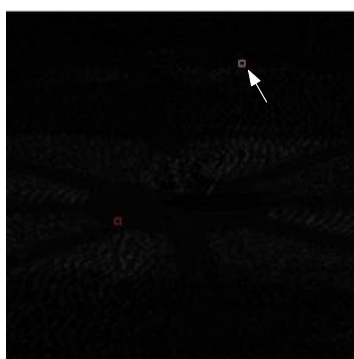

г)

Рисунок 3 - Результаты обработки тестового изображения Square:
a) $\theta=0$; б) $\theta=\pi / 2$; в) $\theta=\pi / 4$; г) $\theta=3 \pi / 4$

На рисунках 4 и 5 представлены результаты для случая, когда блок $\mathrm{R}$ выбран на текстурированной области. Визуальный анализ показывает, что поиск по энергетическим маскам Laws определяет похожий блок достаточно точно.

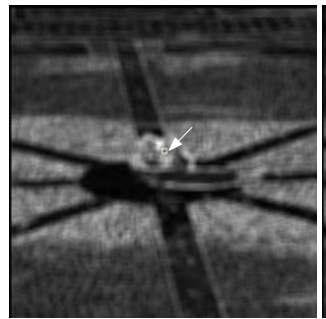

a)

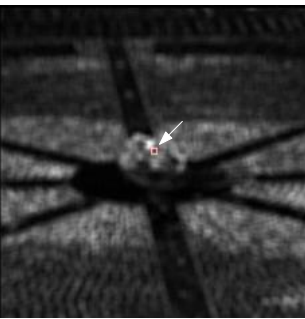

б)

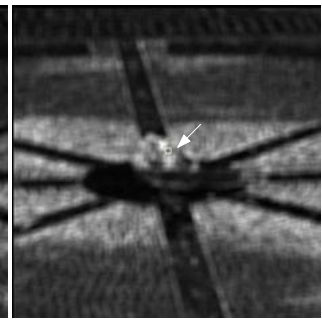

в)

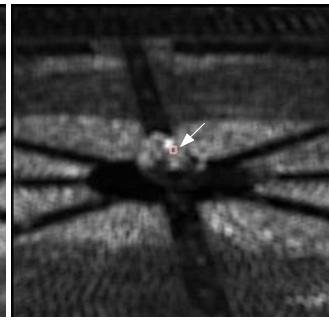

г)

Рисунок 4 - Результаты обработки тестового изображения Square:

a) маска R5W5/W5R; б) маска E5E5; в) маска E5R5/R5E5; г) маска S5S5

Наилучший результат для фильтров Габора получен при применении угла $\theta=0$ (рис. 5а) и $\theta=3 \pi / 4$ (рис. 5в). При $\theta=\pi / 2$ похожий блок найден на границе перепадов яркости (рис. 5б), что является не корректным.

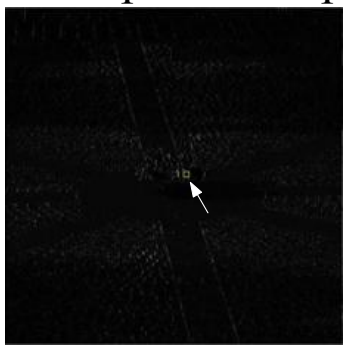

a)

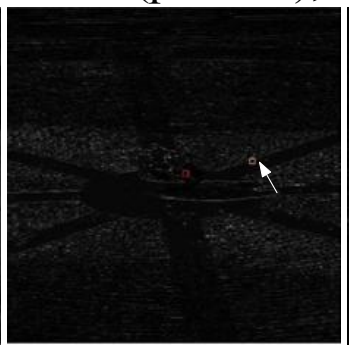

б)

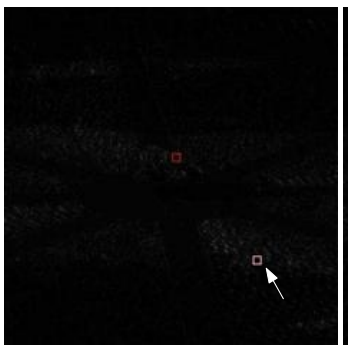

B)

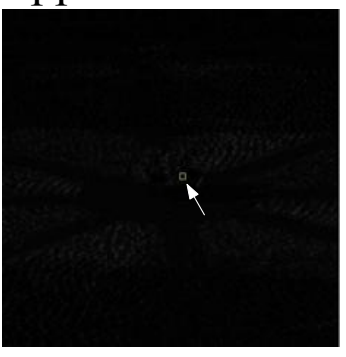

г)

Рисунок 5 - Результаты обработки тестового изображения Square:
a) $\theta=0$; б) $\theta=\pi / 2$
в) $\theta=\pi / 4$; г) $\theta=3 \pi / 4$

Рассмотрим случай, когда блок $\mathrm{R}$ выбран на границе перепадов яркости. Визуальный анализ показывает, что для данного случая на изображениях энергетических характеристик Laws похожий блок определѐн правильно (рис. 6). 


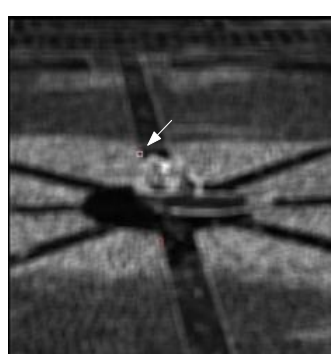

a)

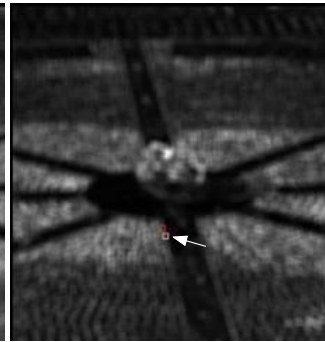

б)

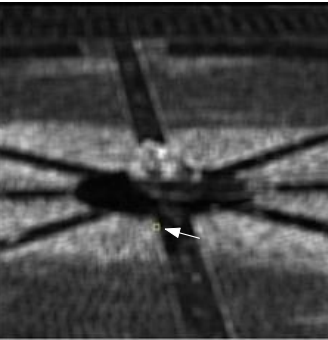

B)

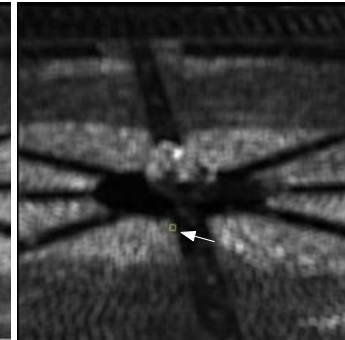

г)

Рисунок 6 - Результаты обработки тестового изображения Square:

a) маска R5W5/W5R; б) маска E5E5; в) маска E5R5/R5E5;

г) маска $\mathrm{S5S5}$

Аналогичный результат был получен для фильтров Габора при угле $\theta=0 \quad$ и $\theta=\pi / 4$ (рис.7). При угле $\theta=\pi / 2$ и $\theta=2 \pi / 4$ похожий блок определен на слабоконтрастной области.

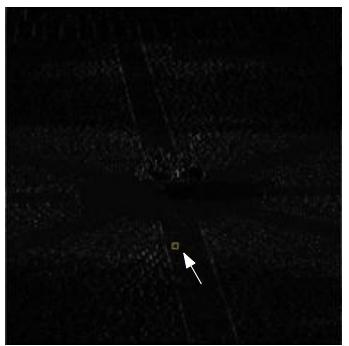

a)

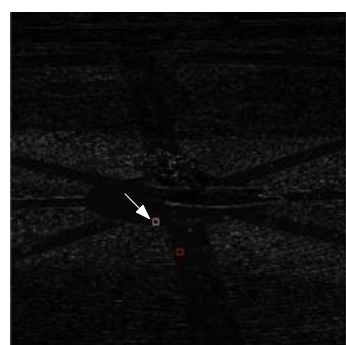

б)

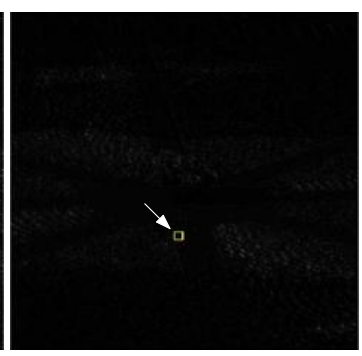

B)

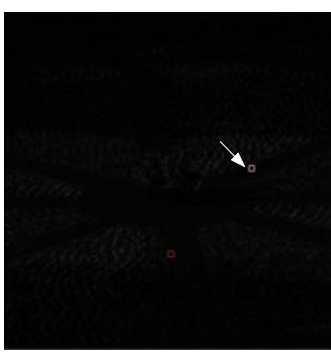

г)

Рисунок 7- Результаты обработки тестового изображения Square:
a) $\theta=0$; б) $\theta=\pi / 2$;
в) $\theta=\pi / 4 ;$ г) $\theta=3 \pi / 4$

Анализ результатов показывает, что использование фильтров Лавса и Габора позволяет получить описание характеристик текстуры в окрестности выбранного пикселя. В будущем предлагается выработать критерий для определения меры похожести на основе текстурных характеристик в сочетании с евклидовой дистанцией.

В заключении можно сделать следующие выводы:

- в работе рассмотрены различные подходы к поиску похожих блоков на изображениях и кадрах видеопоследовательности с использованием текстурного анализа;

- представлен интерес поиска похожих блоков на каждой энергетической карте, с последующей комбинацией найденных блоков для синтеза наиболее похожего блока.

\section{Литература.}

1. Srinivasan G., Shobha G. Statistical Texture Analysis. Proceedings of world academy of science, vol.36, pp.1264-1269, 2008.

2. Гонсалес Р., Вудс Р., Эддинс С. Цифровая обработка изображений в 
среде MATLAB. - М.: Техносфера 2006. - 616 с.

3. Laws K. Textured image segmentation, Ph.D. dissertation, University of Southern California. 130 pp.,1980.

4. Mood, A.; Graybill, F.; Boes, D. Introduction to the Theory of Statistics (3rd ed.). McGraw-Hill. p. 229, 1974. 